\title{
Is the length of esophageal invasion only associated with mediastinal nodal metastasis from adenocarcinoma of the esophagogastric junction (Siewert type II and III) after neo-adjuvant chemoradiotherapy?
}

\author{
Shinji Mine \\ Department of Esophageal and Gastroenterological Surgery, Juntendo University Hospital, Hongo, Bunkyo-ku, Tokyo, Japan \\ Correspondence to: Shinji Mine, MD, PhD. Department of Esophageal and Gastroenterological Surgery, Juntendo University Hospital, 3-1-3 Hongo, \\ Bunkyo-ku, Tokyo 113-8431, Japan. Email: mineshinji921@gmail.com. \\ Provenance: This is an invited article commissioned by the Academic Editor Dr. Shuangjiang Li (Department of Thoracic Surgery and West China \\ Medical Center, West China Hospital, Sichuan University, Chengdu, China). \\ Comment on: Mitchell KG, Ikoma N, Nelson DB, et al. Mediastinal nodal involvement after neoadjuvant chemoradiation for Siewert II/III \\ adenocarcinoma. Ann Thorac Surg 2019;108:845-51.
}

Submitted Sep 05, 2019. Accepted for publication Sep 25, 2019.

doi: $10.21037 /$ jtd.2019.09.78

View this article at: http://dx.doi.org/10.21037/jtd.2019.09.78

I recently read Dr. Mitchell and his/her colleagues' article with great interest (1). They investigated the association between thoracic nodal involvement and clinical factors in patients who underwent surgical resection for Siewert type II or III adenocarcinoma after neoadjuvant chemoradiotherapy. They concluded that thoracic nodal involvement was more strongly associated with the length of esophageal invasion than with the location of the tumor's epicenter (Siewert type classification).

First of all, I would like to appreciate their work because this is the first report on the association between thoracic nodal involvement and the length of esophageal invasion in patients with adenocarcinoma of the esophagogastric junction (AEG) after neoadjuvant chemoradiotherapy, as the authors wrote. I believe that this work will help us to devise a clinical strategy for patients with AEG.

Unfortunately, they did not present the survival data of the patients who had pathologically positive nodes in the mediastinum. If survival of patients with pathologically positive nodes in the mediastinum was very poor, thoracic lymphadenectomy must be omitted. In addition, they did not show the distribution of thoracic nodal involvement. Thus, it is unclear whether these nodes could be resected via a transhiatal approach or only via right thoracotomy.

They defined thoracic nodal involvement as pathologically positive nodes and recurrences in the mediastinum within two years after operation, all together. However, I am afraid that this concept might lead to misunderstanding. It is well known that gastric cancer recurrence after gastrectomy is frequently detected in the abdominal para-aortic nodes (2), as well as some patients with pathologically positive abdominal para-aortic nodes who are cured of gastric cancer after surgical resection (3). A randomized controlled trial was performed to investigate the clinical significance of prophylactic lymphadenectomy of the abdominal para-aortic nodes for patients with gastric cancer in Japan, and no survival benefit was observed (4). Based on these results, I am unable to agree with their concept that the nodes which frequently relapse are clinically important and should be resected.

In their study, only the length of esophageal invasion was independently related to thoracic nodal involvement by multivariate analysis, while Siewert classification was not. I think that this is partly because the number of the patients with Siewert type III were relatively lower in their cohort than that of patients with Siewert type II. Many previous studies showed that Siewert type classification was associated with the distribution of nodal metastasis $(5,6)$. It would be strange if neo-adjuvant treatment changes the distribution of nodal metastasis. 
Another significant problem is the ambiguity of endoscopic findings. In some cases, especially when tumors are large and circumferential, the esophagogastric junction was not detectable by endoscopy, as the authors wrote. However, in such locally advanced cases, the decision whether to perform esophagectomy or gastrectomy is supposed to be very important. It is difficult to clinically determine the length of esophageal invasion as well as Siewert classification in these patients.

The authors concluded that the length of esophageal invasion $\geq 1.5 \mathrm{~cm}$ was significantly related to thoracic nodal involvement. When a patient is diagnosed with Siewert type III AEG and esophageal invasion of $2 \mathrm{~cm}$, should we perform esophagectomy via a right thoracotomy and total gastrectomy?

Nonetheless, I believe that their study will help us to devise treatment strategies for patients with Siewert type II or III. And I think that a prospective study is necessary in the future. Recently, we published a prospective study regarding the distribution of nodal metastasis in the patients with Siewert type II (7), and I expect that it could improve our knowledge of lymphadenectomy.

\section{Acknowledgments}

None.

\section{Footnote}

Conflicts of Interest: The author has no conflicts of interest to declare.

Ethical Statement: The author is accountable for all aspects of the work in ensuring that questions related to the accuracy or integrity of any part of the work are appropriately investigated and resolved.

\section{References}

1. Mitchell KG, Ikoma N, Nelson DB, et al. Mediastinal nodal involvement after neoadjuvant chemoradiation for Siewert II/III adenocarcinoma. Ann Thorac Surg 2019;108:845-51.

2. Baba M, Hokita S, Natsugoe S, et al. Paraaortic lymphadenectomy in patients with advanced carcinoma of the upper-third of the stomach. Hepatogastroenterology 2000;47:893-6.

3. Yonemura $Y$, Segawa $M$, Matsumoto $H$, et al. Surgical results of performing R4 gastrectomy for gastric cancer located in the upper third of the stomach. Surg Today 1994;24:488-93.

4. Sasako M, Sano T, Yamamoto S, et al. D2 lymphadenectomy alone or with para-aortic nodal dissection for gastric cancer. $\mathrm{N}$ Engl J Med 2008;359:453-62.

5. Hosokawa Y, Kinoshita T, Konishi M, et al. Clinicopathological features and prognostic factors of adenocarcinoma of the esophagogastric junction according to Siewert classification: experiences at a single institution in Japan. Ann Surg Oncol 2012;19:677-83.

6. Pedrazzani C, de Manzoni G, Marrelli D, et al. Lymph node involvement in advanced gastroesophageal junction adenocarcinoma. J Thorac Cardiovasc Surg 2007;134:378-85.

7. Kurokawa Y, Takeuchi H, Doki Y, et al. Mapping of lymph node metastasis from esophagogastric junction tumors: a prospective nationwide multicenter study. Ann Surg 2019. [Epub ahead of print].
Cite this article as: Mine S. Is the length of esophageal invasion only associated with mediastinal nodal metastasis from adenocarcinoma of the esophagogastric junction (Siewert type II and III) after neo-adjuvant chemoradiotherapy? J Thorac Dis 2019;11(10):E152-E153. doi: 10.21037/jtd.2019.09.78 